\title{
Mp Tuning for Internal Model Control 2x2 Multi Input Multi Output (MIMO) System
}

\author{
Dinny Winda Astuti ${ }^{1}$ Juwari $^{1}$, and Renanto Handogo ${ }^{1}$
}

\begin{abstract}
IMC is a type of model based control that compensates time delay in the process. The controller tuning is quite simple in case of no-error in the model, otherwise it will be a difficult matter. Mp tuning has been considered a tuning for uncertain processes. To extend IMC to MIMO system, a new method based on Maximum Peak (Mp) is developed. The present study proposes Maximum Peak (Mp) tuning for IMC in 2x2 multi input and multi output (MIMO) system. Three particular 2x2 model of distillation colomn are being studied, the best configuration is analyzed by Relative Gain Array (RGA) and Average Dynamic Gain Array (ADGA) method. The tuning method consists of two main steps: Firstly, determine the worst case of the model uncertainty. Secondly, specify the parameter of set point controller using maximum peak (Mp) criteria. The effectiveness of Mp tuning for IMC in MIMO system is evaluated and compared to Biggest Log Modulus Tuning (BLT) for MIMO-PI Controller, Skogestad Tuning, and Rivera Tuning. Evaluation and comparison have been done through simulation and the results are satisying.
\end{abstract}

Keywords—Maximum Peak, Uncertainty Process, Stability Analysis, Process Interaction.

\section{INTRODUCTION}

$\mathrm{I}_{\mathrm{c} \mathrm{N}}^{\mathrm{C}}$ MC was developed by developed by Morari and coworkers $[5,7,10]$. The philosophy relies on the internal model principle which states that control can be achieved only if the control system encapsulates, either implicitly or explicitly, some representation of the process to be controlled. Internal Model Control uses model explicitly in the controller algorithm. By simply setting $\mathrm{Gc}(\mathrm{s})$ as the inverse of $\mathrm{Gpm}(\mathrm{s})$ and if $\mathrm{Gp}(\mathrm{s})=$ Gpm(s), (the model is an exact representation of the process), then it is clear that the output will always be equal to the set point and this control performance can be achieved without feedback structure. Thus, if complete knowledge encapsulated in the process model, perfect control can be achieved without feedback structure and feedback structure is only necessary when knowledge about the process is inaccurate or incomplete. In practice, difference between process and model is common and usually occurs, the process model may not be invertible and the system is often affected by unknown disturbance. Thus the above open loop control arrangement will not be able to guarantee in maintaining output at setpoint. Nevertheless, it forms the basis for development of a control strategy that has the potential to achieve perfect control. Below is Internal Model Control Structure in Figure 1.

While $G c_{1}$ algorithms are formulated as;

$G c_{1}=\frac{1}{k} \frac{\tau s+1}{\lambda_{1} s+1}$

Where $k$ is gain process, $\tau$ is time constant of the process, $\lambda$ are filter parameter of the controller. The parameters can be easily obtained if no error in the model using Skogestad Tuning or Rivera Tuning. However, if the model contains uncertainty, it will always contain inaccuracies. The model uncertainty may comes from several sources as follows i.e.: the variation of real

${ }^{1}$ Dinny Winda Astuti, Juwari, and Renanto Handogo are with Departement of Chemical Engineering, Faculty of Industrial Technology, Institut Teknologi Sepuluh Nopember, Surabaya, 60111, Indonesia. E-mail: dinny.w.a@gmail.com; joecheits@yahoo.com; pcipro@indo.net. parameters affecting plant operation, the inherent nonlinearity of the processes, the experimental identification of the process and the mathematical model development [11]. In practice the process uncertainty can be obtained from input output test on the plant at different operating points [3]. Recently, Juwari, et all (2012) has developed a new tuning method that employs the stability analysis criteria to obtain parameter for 2DOF-IMC under uncertainty model. This method has been evaluated in FOPDT with $\theta / \tau \leq 1, \theta / \tau \cong 1$ and $\theta / \tau \geq 1$ process, second order with underdamped process, and third order with non-minimum phase systems and yields very satisfying performance in single input and single output system [13]. However, application in multi input and multi output has not been evaluated. In many practical control problems typically a number of variables must be controlled and a number of variables can be controlled. This problems referred to as multiple-input, multiple output (MIMO) control problems. For almost all important processes at least two variables must be controlled. The main characteristic of this system is the presence of process interactions, that is, each manipulated variable can affect all controlled variables [5]. In this paper, the Mp tuning method is applied to IMC for $2 \times 2$ MIMO model. $\lambda$ is set by specifying Mp value. The effectiveness of $\mathrm{Mp}$ tuning method for MIMO system is evaluated and compared with Biggest Log Modulus Tuning (BLT) for MIMO-PI Controller, Skogestad Tuning, and Rivera Tuning for IMC. The output responses for setpoint tracking and disturbance rejection values are compared using Integral Absolute Error (IAE).

\section{A. Multi Input Multi Output Control Systems}

Control systems are classified by their number of controlled variables and manipulated variables. If it consists of more than one controlled variable and one manipulated variable, this system is referred to a multiple input - multiple output (MIMO) system. The main characteristic of MIMO control system is the presence of process interactions, where each of manipulated variable can affect both controlled variables. The number of manipulated variables $(U)$ is equal to the number of controlled variables (Y). MIMO control systems are more complex than single input - 
single output (SISO) systems, because of the presence of process interaction between controlled variables dan manipulated variables. Changes in manipulated variables $U_{1}$, can affect all controlled variables $Y_{1}, Y_{2}, \ldots Y_{n}$. When significant process interactions are present, the selection of the most effective control configuration may not be easy. In particular, for a control problem with $n$ controlled variables and $n$ manipulated variables, there are $n$ ! possible multiloop control configurations [5].Thus, there are two possible configurations for closed-loop control structure in 2 x 2 MIMO system: 1-1/2-2 configuration and 1-2/2-1 configuration. To determine which configuration suits best for the process that is able to minimize process interaction, two wellknown methods are used:

1) Relative Gain Array (RGA)

In RGA method, the interaction is measured between the $i$ th controlled variable and $j$ th controlled variable by parameter $\lambda_{i j}$ (relative gain) which can be calculated using Eq. 2

$$
\lambda=\frac{1}{\frac{K_{12} K_{21}}{K_{11} K_{22}}}
$$

For $2 \times 2$ case, each row and each column of $\lambda$ sums to one, thus the RGA for a 2x2 systems can be expressed as:

$$
\Lambda=\left[\begin{array}{cc}
\lambda & 1-\lambda \\
1-\lambda & \lambda
\end{array}\right]
$$

In RGA approach, if $\lambda$ is close to 0 , the manipulated variable had little effect on the controlled variable, and vice versa if it close to 1 . Thus, the recommended configuration will be the relative gain which is positive and closest to 1 .

2) Average Dynamic Gain Array (ADGA)

The principal of ADGA method is to include the dynamic considerations into the interaction measurement. It was proposed by Gagnepain and Seborg (1982), and formulated as:

$$
\begin{aligned}
& D_{i j}=\frac{1}{\theta-\theta_{1}} \int_{\theta_{1}}^{\theta} y_{i}(t) d t \\
& \mu=D \odot\left[D^{-1}\right]^{T}
\end{aligned}
$$

Where $D_{i j}$ is the average dynamic gain between the manipulated variable and the controlled variable and $D$ is the matrix of element $D_{i j}$ and $\theta_{1}=\min \left\{\max \left(d_{i i}\right), \max \left(d_{i j}\right)\right\}$ and $\theta=\theta_{1}+T_{M}$, where $T_{M}=\max \left(\tau_{i j}\right)$. ADGA's approach is in analog with RGA method that the recommended configuration corresponds the value of average dynamic gain that is positive and closest to 1 .

\section{B. Multi Input Multi Output IMC}

Control design techniques for MIMO systems indicate that good results can be achieved if the system is divided into pairs of input and output variables, and a controller is used to control each of the pair (multiloop strategy). By combining the characteristics of IMC with the multiloop strategy, it will generate a controller design procedure that is simple but effective. Common problems in factorization, inversion, and determination in designing multivariable IMC filter has been investigated in detail by Garcia and Morari (1985) [9]. Computational calculations in the implementation of the $G_{c}$ is highly recommended along with increasing dimensions of the system that must be controlled [10].
To control a MIMO system with IMC is by selecting controller $G_{c}$ and $G_{m}$ process model as a diagonal matrix transfer. Here is the block diagram in Figure 2.

The underlying concept of handling multiloop control systems by IMC controllers is to use the on-diagonal models. However, if only the on-diagonal transfer functions are used, it will certainly lead to a modeling error in the design. Therefore the existing parameter in the filter should be reduced until a stable state is reached, which would make the filter parameters to be the key in designing multivariable IMC. Assuming that the pairing configuration has been determined, then:

$$
G=\left[\begin{array}{ll}
G_{11} & G_{12} \\
G_{21} & G_{22}
\end{array}\right]
$$

So that the process model for multiloop IMC:

$$
G_{m}=\operatorname{diag}\left[G_{11}, G_{22}\right]
$$

And the multiloop IMC controllers:

$G_{c}=\operatorname{diag}\left[G_{c 11}, G_{c 11}\right]$

$$
G_{c i}=G-{ }_{i i} G_{f i} \quad i=1,2, \ldots n
$$

\section{Maximum Peak (Mp)}

Maximum peak $(M p)$ is defined as the maximum magnitude of the closed-loop frequency response. In general, the magnitude of $M p$ considered one of principle of stability analysis which indicates the relative stability of a stable system.

Normally, a large Mp corresponds to a large maximum overshoot of the step response in the time domain [4]. For most control systems, it is generally accepted in practice that the desired $\mathrm{Mp}$ should be in the range between 1 and 1.5 [2]. Low $M p$ value indicates slow control response, while large $M p$ value indicates fast control response and large maximum overshoot which leads to unstability. Brosilow \& Joseph (2001) proposed that the $M p$ value is 1.05 . And with this $M p$ value the overshoot in time response domain will be about $10 \%$.

\section{METHOD}

\section{A. Case Studies}

In this paper, three particular 2x2 MIMO systems which represents laboratory scale distillation columns are studied:

1) Wood \& Berry Column (1973)

$$
G(s)=\left[\begin{array}{cc}
\frac{12.8 e^{-s}}{16.7 s+1} & \frac{-18.9 e^{-3 s}}{21 s+1} \\
\frac{6.6 e^{-7 s}}{10.9 s+1} & \frac{-19.4 e^{-3 s}}{14.4 s+1}
\end{array}\right]
$$

2) Wardle \& Wood Column (1969)

$$
G(s)=\left[\begin{array}{cc}
\frac{0.126 e^{-6 s}}{60 s+1} & \frac{-0.101 e^{-12 s}}{(48 s+1)(45 s+1)} \\
\frac{0.094 e^{-8 s}}{38 s+1} & \frac{-0.12 e^{-8 s}}{35 s+1}
\end{array}\right]
$$


3) Vinante \& Luyben Column (1972)

$$
G(s)=\left[\begin{array}{cc}
\frac{-2.2 e^{-s}}{7 s+1} & \frac{1.3 e^{-0.35 s}}{7 s+1} \\
\frac{-2.8 e^{-1.8 s}}{9.5 s+1} & \frac{4.3 e^{-0.35 s}}{9.2 s+1}
\end{array}\right]
$$

\section{B. Research Procedures}

The research method consists of three steps: (i) to determine the recommended configuration for each $2 \mathrm{x} 2$ control system; (ii) to determine the worst case of each diagonal transfer function (iii) to specify the parameter of controller $(G c)$ using $M p$ criteria based on the worst case as $G p$ and no error in the model as $G p m$.

1) The first step is determining the recommended configuration. Possible configuration for each model will be measured based on the steady state information by RGA method and based on dynamic information by ADGA method. Interaction analysis calculations will be done by MATLAB software.

2) The second step is determining the worst case of diagonal transfer function in each matrix. The worst case can be found from the limit of the uncertainty model in terms of upper and lower on process model parameters. In this paper, the lower and upper limit is $\pm 20 \%$. The worst case can be identified at the biggest maximum value of magnitude of frequency response of complementary sensitivity function. When determining the worst case, time filter constant $(\lambda)$ value is set equal to the time delay of no error in the model.

3) The third step is specifying the parameter of controller $(G c)$ using IMC structure based on the MpTuning criteria as follows;

a. Set $\lambda$ (filter time constant Gc) initial value equal to the time delay $(\theta)$ of process model divided by 100.

b. Because the complementary sensitivity function of MIMO consists of both controllers, to simplify the iteration when determining parameter of each controller, the controller of the other loop is designed as original SISO IMC.

c. Calculate maximum magnitude of closed loop frequency response in the range of frequency $\omega=$ $10^{-3}$ to $10^{3}$.

d. If $\max |T(j \omega)|>1.05$ then increase $\lambda$ by small number, for example $\lambda+0.01$.

To first calculate $|T(j \omega)|$ as SISO IMC, the principle of closed-loop stability of SISO system is used:

$\frac{Y_{1}}{Y_{s p 1}}=\frac{G_{c 1} G_{p 11}}{1+G_{c 1}\left(G_{p 11}-G_{p m 11}\right)}$

$\frac{Y_{2}}{Y_{s p 2}}=\frac{G_{c 2} G_{p 22}}{1+G_{c 2}\left(G_{p 22}-G_{p m 22}\right)}$

To calculate $|T(j \omega)|$ as multiloop IMC that contains the interaction information, principle of closed-loop stability of MIMO system below is used:

$\frac{Y_{1}}{Y_{s p 1}}=\frac{G_{c 1} G_{p 11}+G_{c 1} G_{c 2}\left(G_{p 11} G_{p 22}-G_{p 12} G_{p 21}\right.}{\left.\left(1+G_{c 1} G_{p 11}\right)\left(1+G_{c 2} G p_{22}\right)-G_{c 1} G_{c 2} G_{c 12} G_{c 21}\right)}$
$\frac{Y_{2}}{Y_{s p 2}}=\frac{G_{c 2} G_{p 22}+G_{c 1} G_{c 2}\left(G_{p 11} G_{p 22}-G_{p 12} G_{p 21}\right.}{\left.\left(1+G_{c 2} G_{p 22}\right)\left(1+G_{c 2} G p_{22}\right)-G_{c 1} G_{c 2} G_{c 12} G_{c 21}\right)}$

\section{RESULT AND DISCUSSION}

\section{A. Interaction Analysis}

After conducting interaction analysis for all column, the results in Table 1 is a recommended pairing by RGA and ADGA methods, both RGA and ADGA recommends the same configuration for all column that is $1-1 / 2-2$. This means that the first controlled variable is paired to the first manipulated variable and the second controlled variable is paired to the second manipulated variable.

\section{B. Parameter Setting}

To evaluate the performance of the proposed method, the closed-loop responses were compared to several well-known methods such as biggest log modulus tuning (BLT) for MIMO - PI controller, Skogestad IMC Tuning and Rivera IMC - Tuning. Simulation studies are conducted to illustrate the effectiveness of the Mp-GM method in MIMO IMC. The method is carried out to three particular 2x2 case: Wood \& Berry, Wardle \& Wood and Vinante \& Luyben column. The results are compared to the above well-known tuning method. For SISO IMC - Mp tuning, the complementary sensitivity function is calculated by assuming singleloop-only for each controller. This is essential to calculate MIMO IMC - Tuning since the complementary sensitivity function of 2 × 2 MIMO for loop 1 and loop 2 includes both controller. Parameter of controller for each loop is set so that the complementary sensitivity response of each loop has maximum value of 1.05 (see Fig. 5-10) which corresponds to $10 \%$ overshoot. By having $10 \%$ overshoot, hopefully the response is neither to sluggish nor too sensitive which leads to instability.

In the simulation study, step changes are introduced for both loops and disturbance is induced later in the first loop.

From Fig. 11 to 16 and Table 3, it can be concluded that the proposed method provides a sastisfying response. It even can provide smoother overshoot eventhough not exactly $10 \%$. This might be caused by interaction that occurs in MIMO system, the presence of interaction affects the stability even when calculation of complementary sensitivity function already accounted for it.

\section{CONCLUSION}

In this paper, we proposed an alternative method of designing multi-loop IMC controllers. The proposed method applies one of stability analysis principle to optimizes system stability in 2x2 MIMO systems espescially if there are process uncertainty. Using the property of frequency-dependent property in the closedloop interactions, controller parameter is set so that it can give smoother response with no higher overshoot nor sluggish response. Since practically, the model cannot exactly represents the process using maximum magnitude of closed-loop frequency response to value 1.05 , the response is satisfying in comparison with several well-known tuning methods. 


\section{REFERENCES}

[1]. Luyben, W. L, (1989), "Process Modelling Simulation and Control for Chemical Engineers”, 2nd ed., McGraw-Hill, Inc., New York.

[2]. Kuo, B.C., “Automatic control systems”, 7th ed. Englewood Cliffs, New Jersey: Prentice Hall (1995)

[3]. Brosilow, C. and Joseph, B., "Techniques of model-based control”, Prentice Hall International Series in the Physical and Chemical Engineering Science. New Jersey: Prentice Hall PTR (2001).

[4]. Levine, W.S., "The control handbook", Boca Raton: CRC Press (1995).

[5]. Seborg, D.E., Edgar, T.F. and Mellicamp, D.A., "Process dynamic and control”, 3rd ed. USA: John Wiley \& Sons, Inc (2011).

[6]. Garcia, C.E. and Morari, M., "Internal model control. A unifying review and some new results”, Ind. \& Eng. Chem. Pro. Des. and Dev. 21(2): 308-323 (1982).

[7]. Gagnepain D. E and Seborg, (1982), "Analysis of Process Interaction with Application to Multiloop Control System Design”, IEC. Process Des. Dev. 21, 5-13.

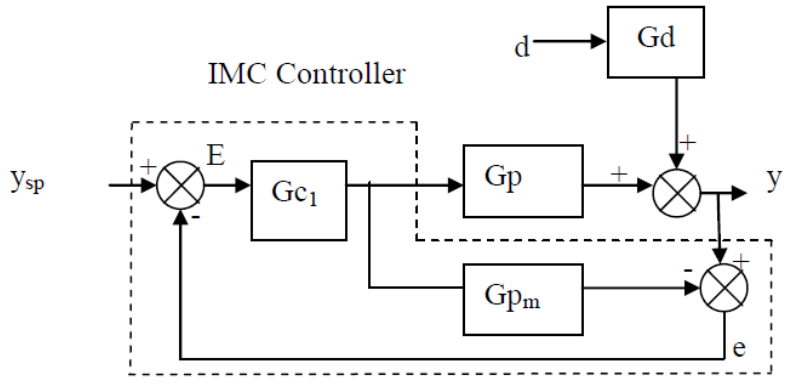

Figure 1. Internal Model Control Scheme

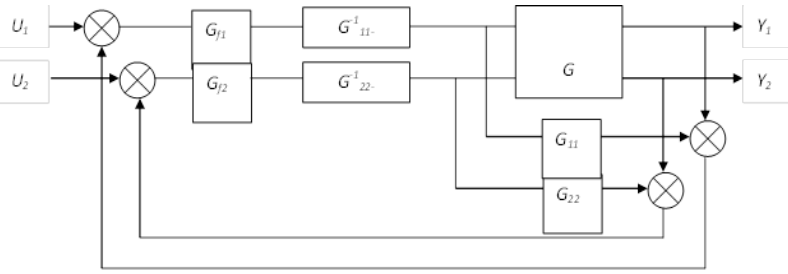

Figure 2. Block diagram of 2 x 2 MIMO IMC Structure

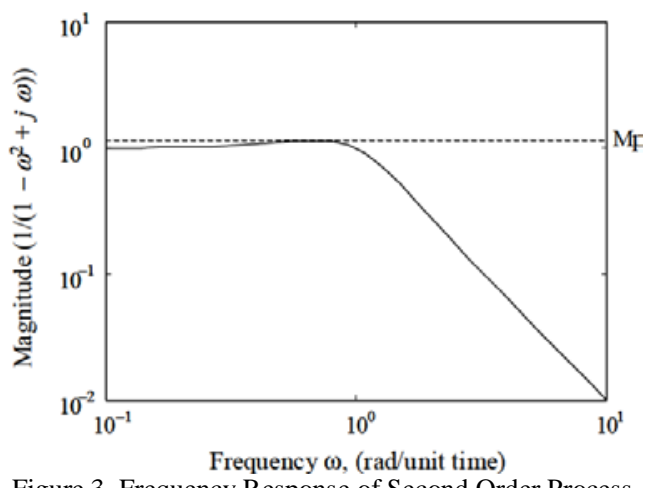

Figure 3. Frequency Response of Second Order Process

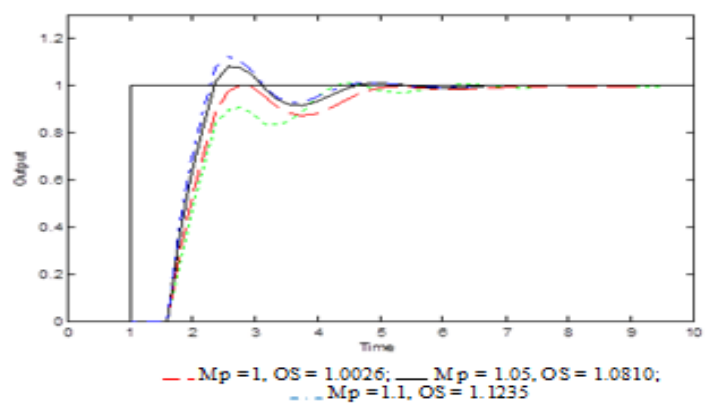

Figure 4. Correlation of Mp value to maximum overshoot in step response
[8]. Rivera, D.E., Morari, M. and Skogestad, S., "Internal model control: PID controller design”, Ind. \& Eng. Chem. Pro. Des. and Dev.. 25(1): 252-265 (1986).

[9]. Garcia, C. E. and Morari M., (1985), "Internal Model Control: Design Procedure for Multivariable Systems”, Ind \& Eng. Chem. Pro. Des. and Dev. 24, 472-484.

[10]. Economou C. G. And Morari M., (1986), "Interbal Model Control: Multiloop Design”, Ind. \& Eng. Chem. Pro. Des. and Dev. 25, 411-419.

[11]. Laughlin, D.L., Jordan, K.G. and Morari, M., "Internal model control and process uncertainty : mapping uncertainty regions for SISO controller design”, Int. J of Cont. 44(6): 1675-1698 (1986).

[12]. Morari, M. and Zafiriou, E., "Robust process control", Englewood Cliffs, NJ: Prentice -Hall (1989).

[13]. Juwari, Badhrulhisham AA., Yee, C. S., and Rosbi M., (2013), “A New Tuning Method for Two-Degree-ofFreedom Internal Model Control under Parametric Uncertainty”, CJCE Vol 21, 1030-1037.

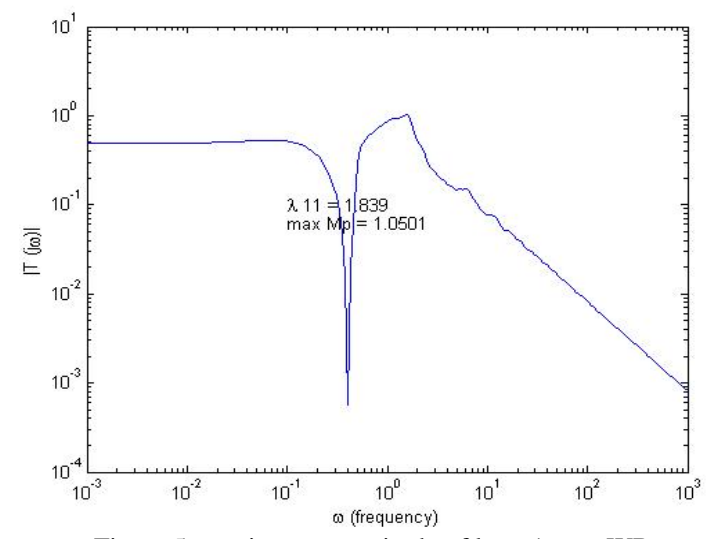

Figure 5. maximum magnitude of loop 1 case WB

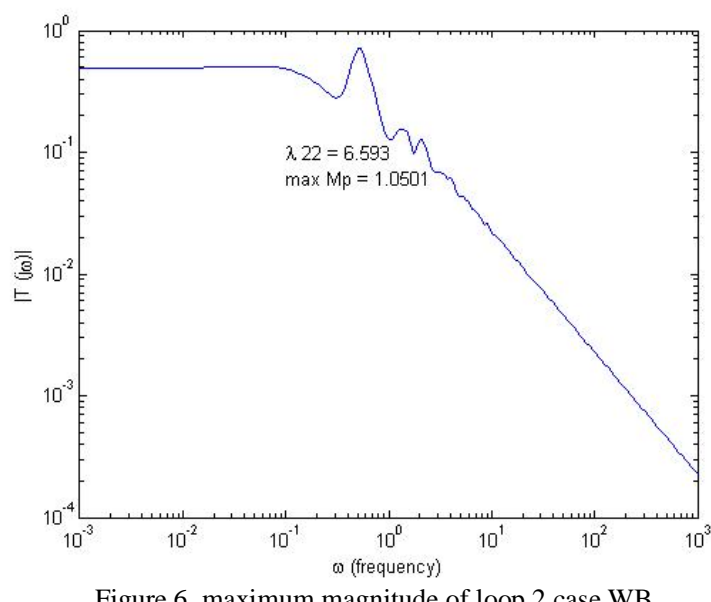

Figure 6. maximum magnitude of loop 2 case WB

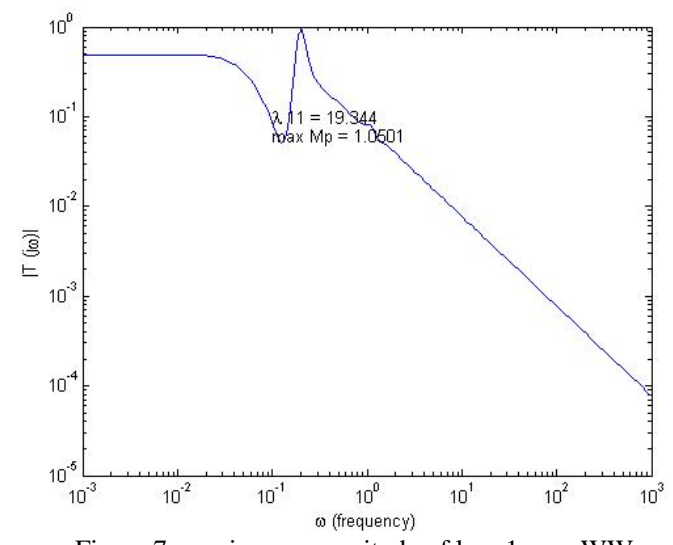

Figure 7. maximum magnitude of loop1 case WW 


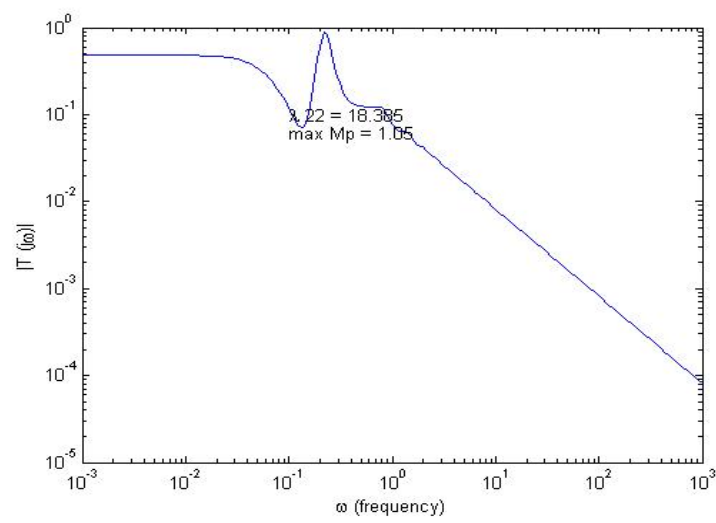

Figure 8. maximum magnitude of loop 2 case WW

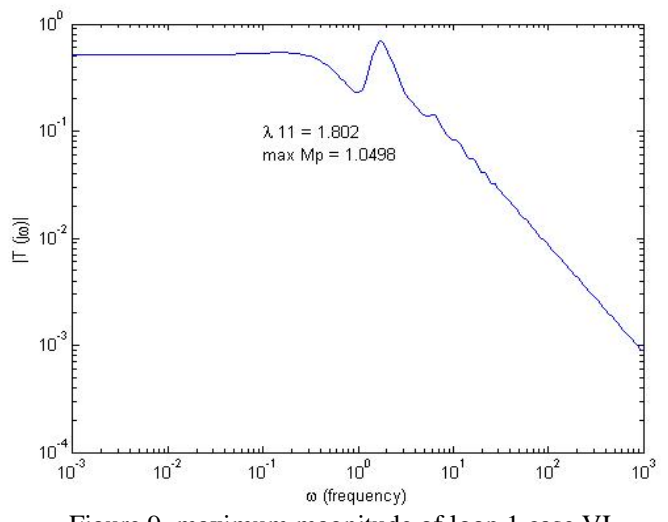

Figure 9. maximum magnitude of loop 1 case VL

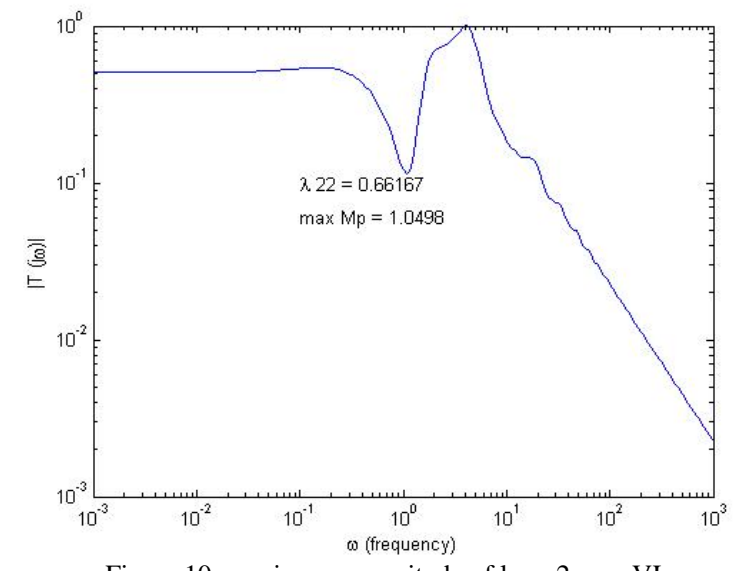

Figure 10. maximum magnitude of loop 2 case VL

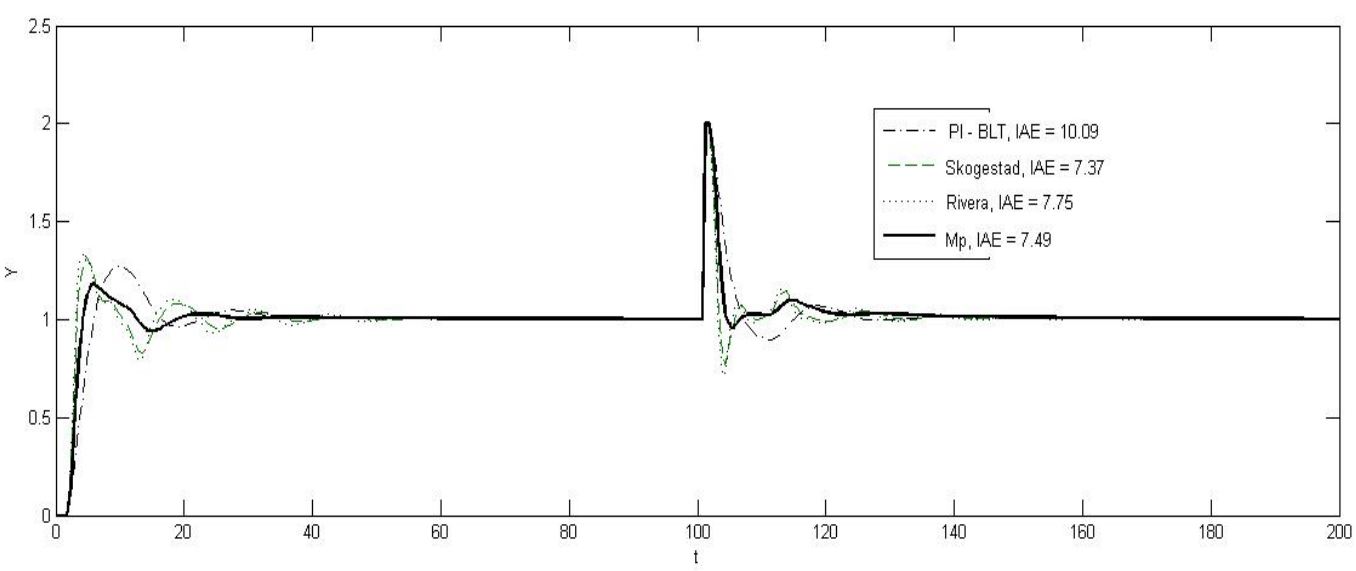

Figure. 11. Closed-loop responses of loop 1 case WB in various tuning

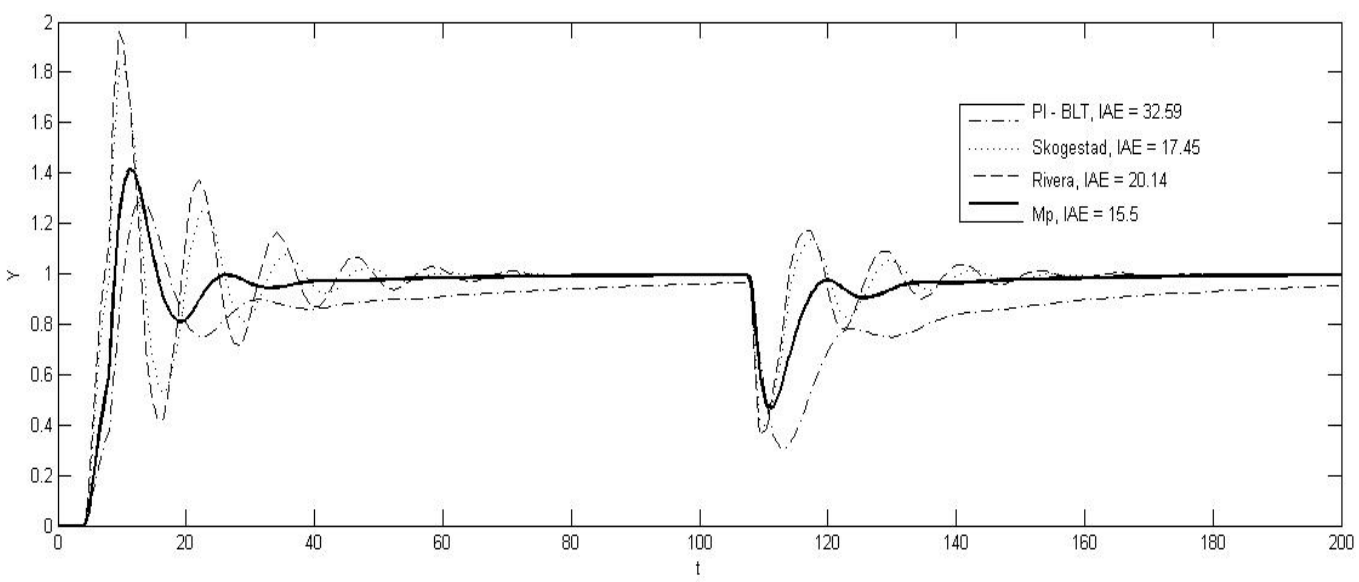

Figure. 12. Closed-loop responses of loop 1 case WB in various tuning 


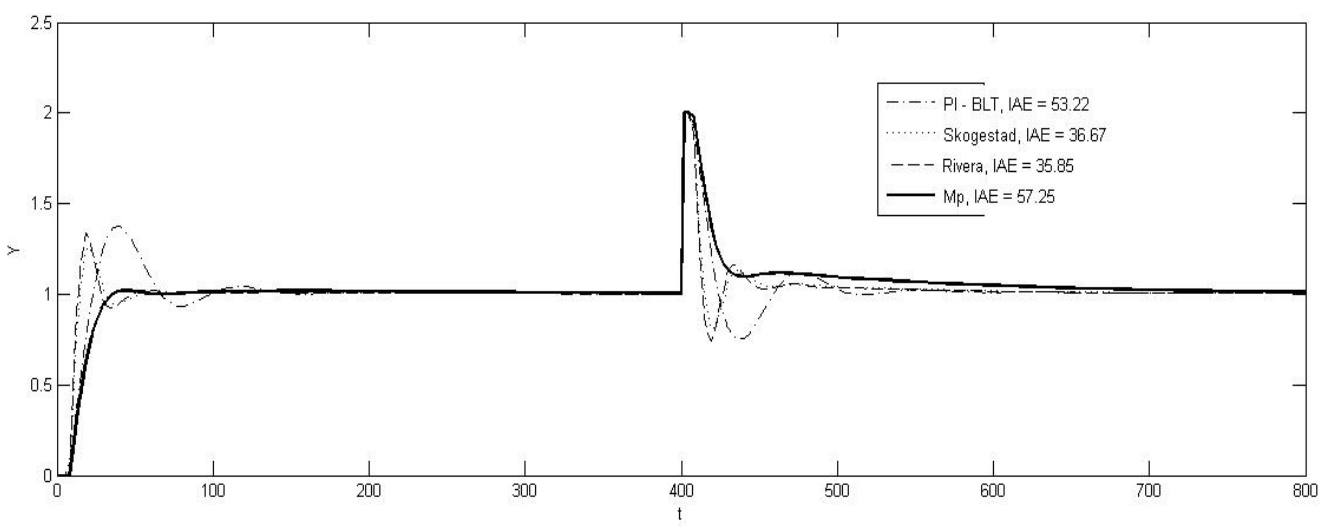

Figure. 13. Closed-loop responses of loop 1 case WW in various tuning

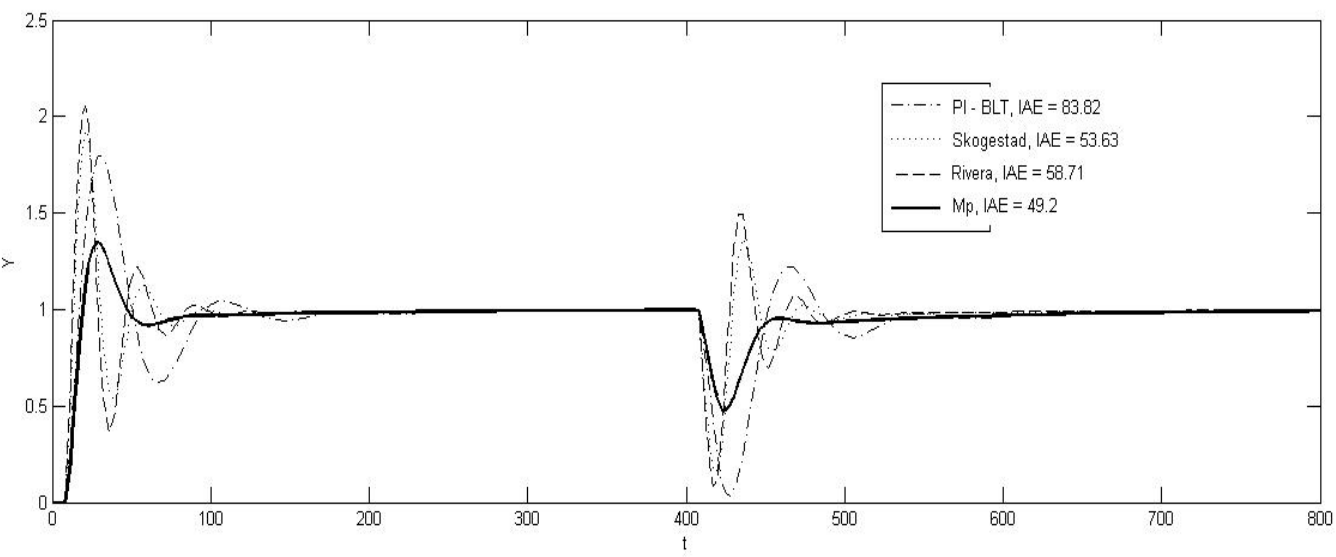

Figure. 14. Closed-loop responses of loop 2 case WW in various tuning

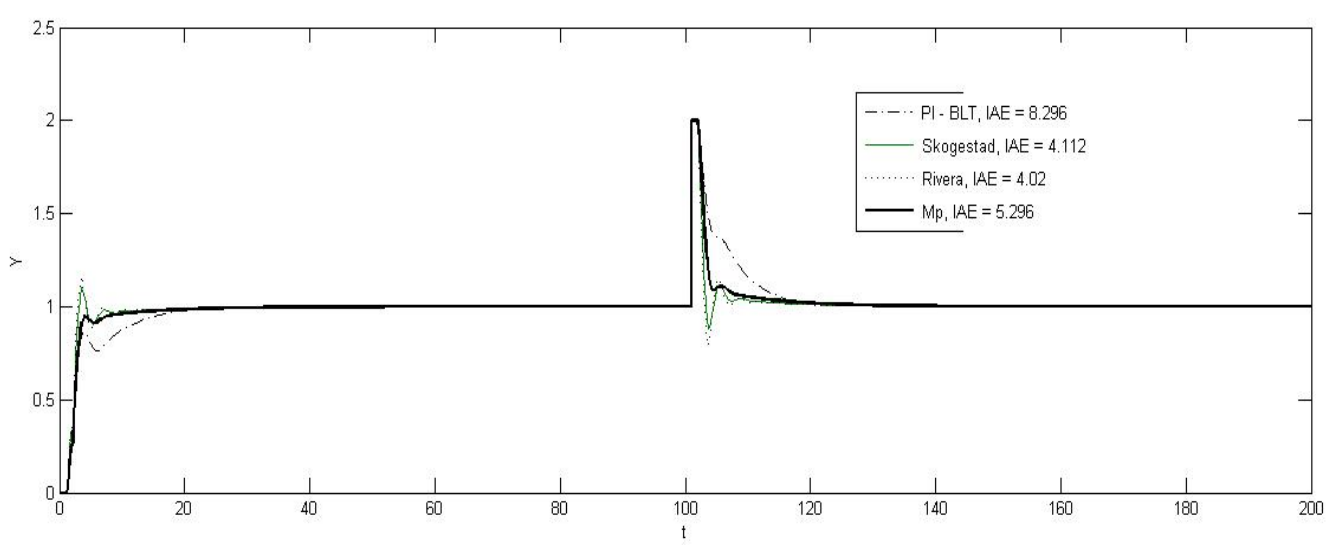

Figure. 15. Closed-loop responses of loop 1 case VL in various tuning

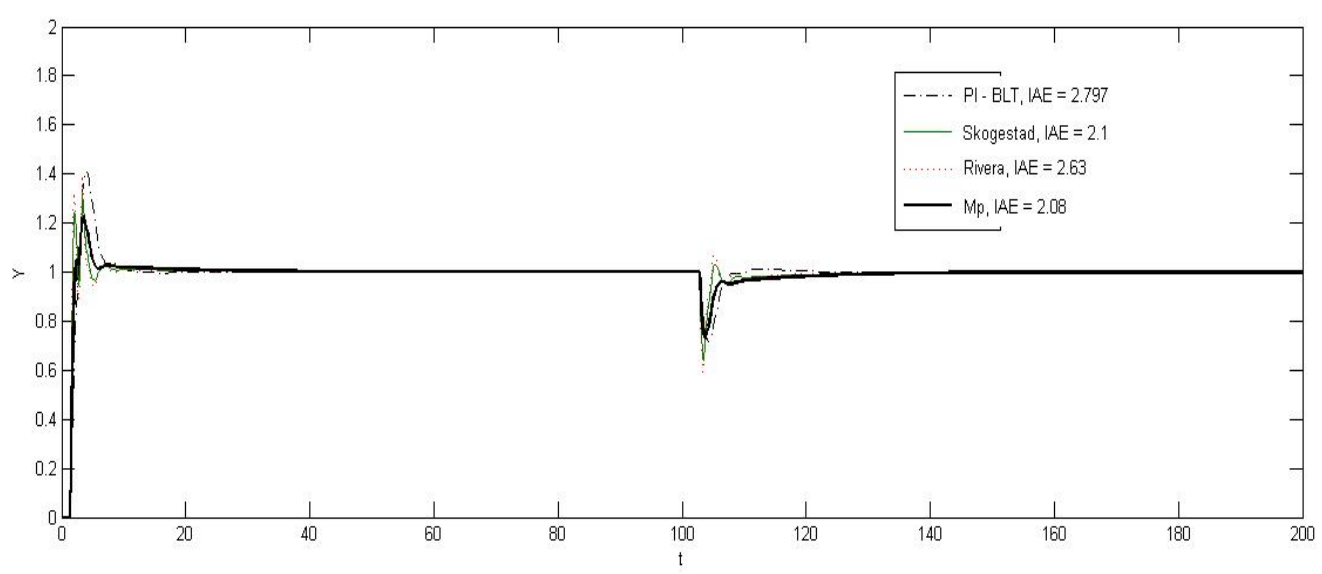

Figure. 16. Closed-loop responses of loop 2 case VL in various tuning 
TABLE 1.

RESULTS OF INTERACTION ANALYSIS

\begin{tabular}{|c|c|c|c|c|c|}
\hline Case & RGA & & ADGA & & Pairing \\
\hline WB & {$\left[\begin{array}{l}2,0094 \\
-1,0094\end{array}\right.$} & $\left.\begin{array}{r}-1,0094 \\
2,0094\end{array}\right]$ & {$\left[\begin{array}{l}1,332 \\
-0,332\end{array}\right.$} & $\left.\begin{array}{r}-0,332 \\
1,332\end{array}\right]$ & $1-1 / 2-2$ \\
\hline WW & {$\left[\begin{array}{l}2,6875 \\
-1,6875\end{array}\right.$} & $\left.\begin{array}{r}-1,6875 \\
2,6875\end{array}\right]$ & {$\left[\begin{array}{l}2,383 \\
-1,383\end{array}\right.$} & $\left.\begin{array}{r}-1,383 \\
2,383\end{array}\right]$ & $1-1 / 2-2$ \\
\hline VL & {$\left[\begin{array}{l}1,6254 \\
-0,6254\end{array}\right.$} & $\left.\begin{array}{c}-0,6254 \\
1,62544\end{array}\right]$ & {$\left[\begin{array}{l}1,210 \\
-0,210\end{array}\right.$} & $\left.\begin{array}{r}-0,210 \\
1,210\end{array}\right]$ & $1-1 / 2-2$ \\
\hline
\end{tabular}

TABLE 2.

CONTROLLER PARAMETER

\begin{tabular}{|c|c|c|c|c|c|c|c|}
\hline & \multirow[t]{2}{*}{ Case } & \multicolumn{2}{|c|}{ PI - BLT Tuning } & \multirow{2}{*}{$\begin{array}{c}\text { IMC Skogestad Tuning } \\
\lambda=\theta\end{array}$} & \multirow{2}{*}{$\begin{array}{c}\text { IMC Rivera Tuning } \\
\lambda=0.8 \theta\end{array}$} & $\begin{array}{l}\text { SISO IMC }-\mathrm{Mp} \\
\text { Tuning }\end{array}$ & $\begin{array}{l}\text { MIMO IMC - Mp } \\
\text { Tuning }\end{array}$ \\
\hline & & $K c_{B L T}$ & $\tau_{i B L T}$ & & & $\lambda$ & $\lambda$ \\
\hline \multirow[t]{2}{*}{ WB } & Loop1 & 0.37 & 8.23 & 1 & 0.8 & 1.611 & 1.839 \\
\hline & Loop2 & -0.07 & 23.46 & 3 & 2.4 & 4.359 & 6.593 \\
\hline \multirow[t]{2}{*}{ WW } & Loop1 & 27.88 & 40.33 & 6 & 4.8 & 9.363 & 19.344 \\
\hline & Loop2 & -13.47 & 41.47 & 8 & 6.4 & 12.377 & 18.385 \\
\hline \multirow[t]{2}{*}{ VL } & Loop1 & 1.07 & 7.06 & 1 & 0.8 & 1.620 & 1.802 \\
\hline & Loop2 & 1.96 & 2.57 & 0.35 & 0.28 & 0.578 & 0.662 \\
\hline
\end{tabular}

TABLE 3.

IAE COMPARISON USING VARIOUS METHOD

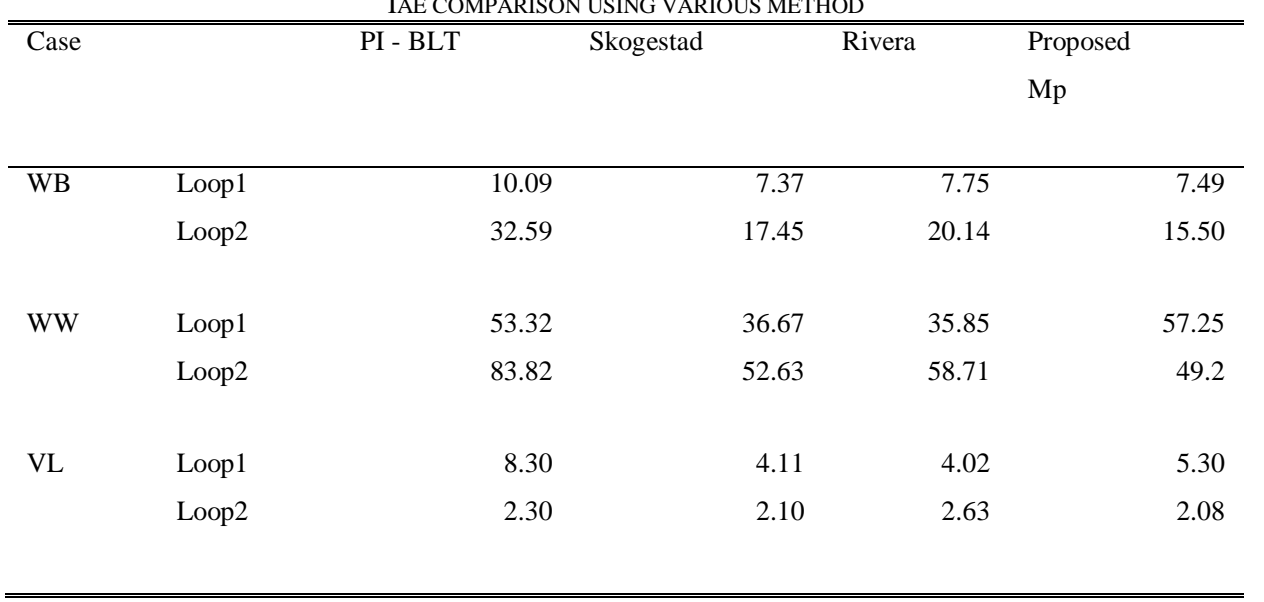

